\title{
Abdominal muscle paresis due to herpes zoster with hiatal hernia - A case report -
}

Received June 28, 2017

Revised 1st, July 19, 2017

2nd, August 1, 2017

3rd, August 4, 2017

Accepted August 7, 2017

\section{Corresponding author}

Ji-Hye Lee, M.D.

Department of Anesthesiology and

Pain Medicine, Presbyterian Medical

Center, 365 Seowon-ro, Wansan-gu,

Jeonju 54987, Korea

Tel: 82-63-230-1595

Fax: 82-63-230-1599

E-mail: 02ljh@hanmail.net

ORCID

http://orcid.org/0000-0003-3269-3844

\author{
Ji-Hye Lee, Hyun-joo Heo, Ikthae Kim, and Sung In Shin \\ Department of Anesthesiology and Pain Medicine, Presbyterian Medical Center, Jeonju, \\ Korea
}

\begin{abstract}
Herpes zoster is an infectious disease of the dorsal root ganglion and sensory nerve fibers. Motor nerve involvement is a rare complication. Hiatal hernia occurs when the stomach protrudes into the thoracic cavity through the esophageal hiatus of the diaphragm. Herein, we present the case of a 78-year-old man with abdominal wall muscle paresis caused by herpes zoster, which resulted in a hiatal hernia.
\end{abstract}

Key Words: Abdominal muscles, Herpes zoster, Hiatal hernia, Paresis.
Herpes zoster (HZ), or shingles, is an infectious disease characterized by the reactivation of varicella-zoster virus that is latent in the dorsal root ganglion of a nerve. HZ typically presents as skin lesions that may take the form of unilateral vesicles or an eschar with pain and sensory disturbances from a single dorsal root. Motor nerve involvement is less common. Motor complications sometimes occur in the segments corresponding to the involved sensory dermatomes. According to studies, about $5 \%$ to $15 \%$ of patients with $\mathrm{HZ}$ have complications involving muscle atrophy and motor paresis due to motor neuron abnormalities $[1,2]$.

Hiatal hernia is a condition in which the gastrointestinal tract is elevated above the diaphragm. The cause of hiatal hernia is not clear [3].

We report a case of abdominal wall muscle paralysis with a hiatal hernia due to $\mathrm{HZ}$.

\section{CASE REPORT}

A 78-year-old man visited our emergency care center following the onset of pain 4-5 days earlier, followed by the dis- covery of a rash and a vesicle on the left side of his abdomen one day prior to his presentation.

Upon his first visit to our emergency care center, the patient was alert. His blood pressure was 140/70 $\mathrm{mmHg}$, heart rate was 50 beats/min, respiratory rate was 20 times $/ \mathrm{min}$, and body temperature was $36.7^{\circ} \mathrm{C}$. His height and weight were $159.7 \mathrm{~cm}$ and $62 \mathrm{~kg}$. In the emergency room, the patient was doubled over and clenching his abdomen due to pain, and rated his level of pain as 60-70 on a visual analogue scale (VAS) of 100. The results of laboratory tests for white blood cells, hemoglobin, platelets, liver function, electrolytes, renal function, calcium, and thyroid function were all normal. However, C-reactive protein was increased to $2.52 \mathrm{mg} / \mathrm{dl}$. The patient had previously been undergoing internal medicine treatment at our hospital. His medical history included hypertension, heart failure, and chronic obstructive pulmonary disease. He had no previous history of diabetes or gastrointestinal symptoms or disorders. He also had no history of trauma or surgery.

On physical examination, the left side of the patient's abdomen in the dermatomes of the 8th to 11th thoracic nerves

This is an Open Access article distributed under the terms of the Creative Commons Attribution Non-Commercial License (http://creativecommons.org/licenses/by-nc/4.0) which permits unrestricted non-commercial use, distribution, and reproduction in any medium, provided the original work is properly cited.

Copyright (C) the Korean Society of Anesthesiologists, 2018 
showed rash, vesicular eruptions, and a marked bulging appearance (Fig. 1). The bulging became more pronounced with increasing abdominal pressure. Bowel sounds were normal. There was no evidence of bowel protrusion into a hernia sac, and no abnormalities other than the bulging were noted.

Abdominal computed tomography (CT) with contrast enhancement revealed no soft tissue abscesses or masses, but a mild sliding type hiatal gastric hernia was visible, along with a right renal stone. There were no mass lesions in or around the spinal canal. Comparison of the CT images with those from studies performed during a previous visit to the internal medicine department revealed that the hiatal gastric hernia was a new finding (Fig. 2). Electromyography (EMG) showed a sharp positive wave and a fibrillation potential on the left external oblique muscle, which was the site of the abdominal bulge on the trunk. These EMG findings denoted a motor

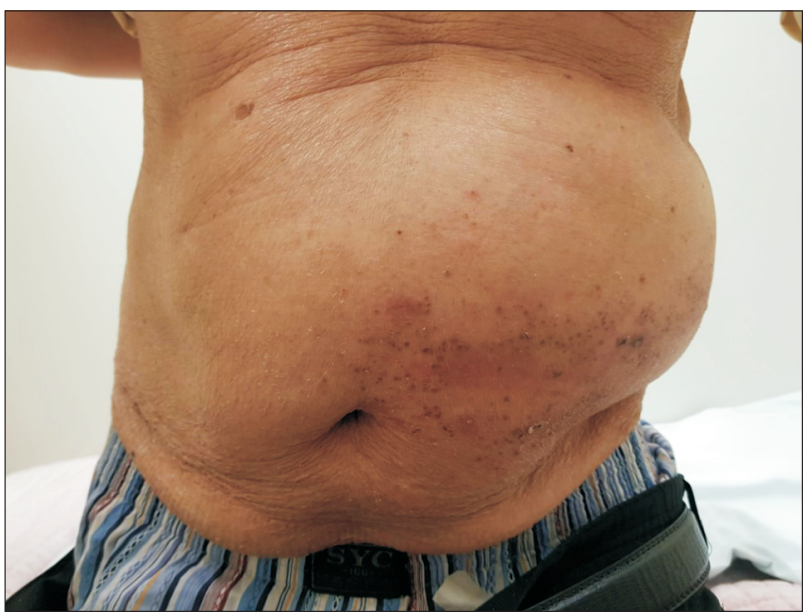

Fig. 1. Bulging appearance of the left flank. neuropathy.

The patient was diagnosed with HZ based on the presence of the typical characteristic signs and symptoms of the condition, such as a segmental distribution of vesicular eruptions and pain. Upon examination, findings consistent with diabetes were not observed, and the use of abdominal CT allowed the exclusion of diseases affecting the spinal cord or roots such as lumbar disk hernia, syringomyelia, and spinal tumors. The patient was treated with oral antivirals, analgesics, anticonvulsants, and nerve blocks. The hiatal hernia was closely observed, and symptomatic treatments were discussed with the internal medicine and surgical departments. As the patient reported experiencing a burning sensation and uncomfortable feelings, he was prescribed a proton pump inhibitor. This led to a reduction in pain that was measured as a VAS score of 20-30, but the abdominal wall muscle paralysis was still visible. Abdominal discomfort, such as the sensation of heartburn, was reduced.

The patient underwent follow-up for one month, and the paralysis of the abdominal wall muscle was reduced by about $50 \%$ compared to its initial state. The patient's pain was reduced to a VAS score of 10-20, and he reported no abdominal discomfort.

\section{DISCUSSION}

In general, $\mathrm{HZ}$ that involves the dorsal root ganglion can damage sensory neurons. Its characteristic symptoms include an erythematous skin rash and painful sensory changes in the affected dermatomes. Although it is rare, involvement of motor or visceral nerves innervating the similar segments
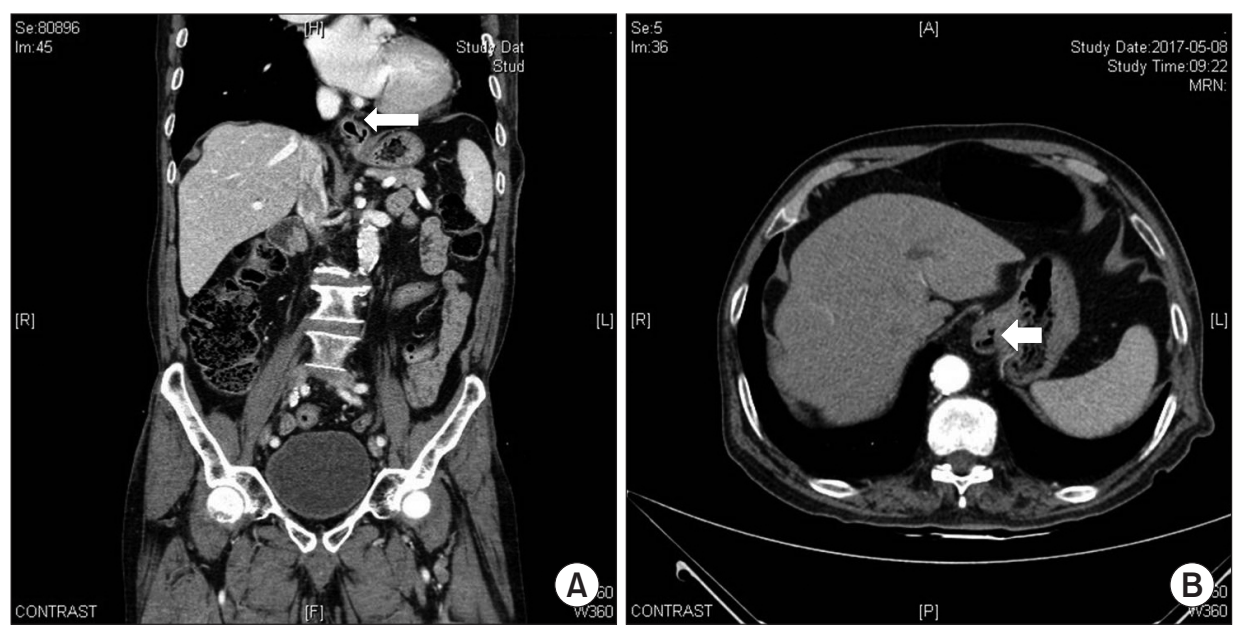

Fig. 2. (A) Coronal and (B) axial enhanced abdominal computed tomography showing a mild sliding type hiatal gastric hernia (arrows). 
of the involved sensory dermatomes can also occur in HZ.

In 1866, Broadbent [4] first reported a case of paralysis owing to motor neuropathy caused by HZ. The rate of motor complications involving muscle atrophy and motor paresis associated with $\mathrm{HZ}$ is approximately $5 \%$ to $15 \%$ [1,2]. Furthermore, studies that have examined motor complications have shown that $25 \%$ of cases exhibited paralysis of muscles innervated by cranial nerves (such as in Ramsay Hunt syndrome); $5.0 \%$ showed segmental paresis of the limbs; $0.75 \%$ had visceral paralysis (involving the gastrointestinal and urinary tract, which resulted in colonic pseudo-obstruction, constipation, or bladder dysfunction); and $0.40 \%$ had paralysis of the diaphragm or abdominal muscles $[2,5]$.

The abdominal muscles (external and internal oblique, transverse abdominal, and rectus abdominis) are innervated by the T7-L1 segmental nerve roots. Thoracic dermatomes are the most commonly affected sites in HZ, and paresis of the abdominal muscles has been infrequently reported. The most commonly affected dermatome in cases of abdominal paralysis was T11 [6-8]. Paralysis of the abdominal muscles may occur in the abdomen, resulting in pseudohernia. In the present case, it was possible to identify the paralysis with the use of an imaging study (Fig. 1). The precise pathogenetic mechanism of muscle paralysis following $\mathrm{HZ}$ is imperfectly understood. The inflammation caused by the virus originating from the dorsal root ganglion or the spinal cord (the anterior horn or ventral root) is explained by its progression to the motor neurons or the secondary degeneration of the involved motor neurons. In addition, inflammation along the nerve fibers can occur as the process progresses.

The esophageal hiatus is an opening in the muscle of the right crus of the diaphragm for the esophagus and the vagus nerve to pass through. The esophageal hiatus is anatomically located at the 10th thoracic vertebral level. The right crus is larger and longer than the left crus. A sliding hiatal hernia is a condition in which the stomach bulges up into the thoracic cavity through the esophageal hiatus. This is the more common type of hernia. In most cases, a hiatal hernia is caused by an increase in abdominal pressure. Acquired hernia through the esophageal hiatus is more common $[3,9]$. The patient in the present case did not report any abdominal discomfort prior to the manifestation of abdominal wall muscle paresis due to HZ, and hiatal hernia was not observed on plain radiographs or chest CT. However, when the patient arrived at the emergency care center, he was compressing his abdomen and raising his legs owing to pain. It is thought that such a posture increased the abdominal pressure and created a hiatal hernia. Even though abdominal muscle paralysis was present, it did not seem to have prevented the increase in abdominal pressure.

Treatment goals of $\mathrm{HZ}$ are to relieve acute pain and prevent postherpetic neuralgia. Antiviral agents that inhibit viral replication have been shown to reduce the duration of viral shedding, hasten rash healing, and decrease the severity and duration of acute pain. Simple analgesics, corticosteroids, opioids, anticonvulsants, tricyclic antidepressants, and neural blockade have been investigated or considered as strategies to achieve these goals. Most patients (55\%-75\%) with motor paralysis due to $\mathrm{HZ}$ are known to recover within 3 months to one year of the onset, but around $20 \%$ of patients recover only partially or remain paralyzed $[2,6-8]$.

Treatment for hiatal hernia is preceded by observation of symptoms. Most people do not experience any symptoms in association with a hiatal hernia, so treatment is unnecessary. Other symptoms that may occur along with a hernia, such as chest pain should be properly evaluated. Symptoms of gastroesophageal reflux disease (GERD), such as heartburn, should be treated, and surgical treatment may be considered if the symptoms do not improve [3,9]. Initial treatment for symptoms of GERD begins with lifestyle modifications such as not wearing tight belts or clothing that can increase the pressure on the abdomen, and taking medication for GERD.

This case was presented to increase awareness of hiatal hernia and motor paralysis in $\mathrm{HZ}$, and the benign and selflimited nature of these conditions. Most hiatal hernias rarely need specific treatment. However, caution is required to avoid the misdiagnosis and treatment of abdominal wall paresis as hernia with sac and hiatal hernia.

Recognition of this complication in patients with $\mathrm{HZ}$ is important, because it is a potentially reversible condition with a good prognosis and does not require surgical intervention.

\section{REFERENCES}

1. Dworkin RH, Johnson RW, Breuer J, Gnann JW, Levin MJ, Backonja $\mathrm{M}$, et al. Recommendations for the management of herpes zoster. Clin Infect Dis 2007; 44 Suppl 1: S1-26.

2. Weiss S, Streifler M, Weiser HJ. Motor lesions in herpes zoster. 
Incidence and special features. Eur Neurol 1975; 13: 332-8.

3. Roman S, Kahrilas PJ. The diagnosis and management of hiatus hernia. BMJ 2014; 349: g6154.

4. Broadbent $\mathrm{WH}$. Case of herpetic eruption in the course of branches of the brachial plexus, followed by partial paralysis in corresponding motor nerves. Br Med J 1866; 2: 460.

5. Maeda K, Furukawa K, Sanada M, Kawai H, Yasuda H. Constipation and segmental abdominal paresis followed by herpes zoster. Intern Med 2007; 46: 1487-8.

6. Chernev I, Dado D. Segmental zoster abdominal paresis (zoster pseudohernia): a review of the literature. PM R 2013; 5: 786-90.

7. Lim HK, Park JH, Lee KH. Herpes zoster induced pure motor segmental paresis: a case report. Anesth Pain Med 2012; 7: 210-2.

8. Kim YY, Kim YS, Park SH. Diagnosis of diaphragmatic paralysis using ultrasound in a cervical herpes zoster patient: a case report. Anesth Pain Med 2016; 11: 76-9.

9. Kohn GP, Price RR, DeMeester SR, Zehetner J, Muensterer OJ, Awad Z, et al. Guidelines for the management of hiatal hernia. Surg Endosc 2013; 27: 4409-28. 